\title{
Modelos teóricos para indicação e implementação de tecnologia assistiva ${ }^{1}$
}

\author{
Ana Cristina de Jesus Alves ${ }^{a}$, Thelma Simóes Matsukura ${ }^{b}$ \\ ${ }^{a}$ Departamento de Terapia Ocupacional, Faculdade de Ceilândia, Universidade de Brasília - UnB, \\ Brasília, DF, Brasil. \\ 'bepartamento de Terapia Ocupacional, Universidade Federal de São Carlos - UFSCar, São Carlos, SP, Brasil.
}

\begin{abstract}
Resumo: Introdução: Pesquisas internacionais buscam compreender os fatores que influenciam o uso bem sucedido dos dispositivos de tecnologia assistiva (T.A.), seja por meio de estudos de sistematização de avaliações, de causas de abandono dos dispositivos ou de modelos teóricos que abordam aspectos necessários à implementação dos mesmos. No Brasil, as pesquisas estão focadas no desenvolvimento de novas tecnologias, sendo ainda escassos os estudos sobre o uso bem sucedido dos dispositivos e as formas de implementação de T.A. Objetivo: Identificar os modelos conceituais mais utilizados para a indicação e a implementação de dispositivos de tecnologia assistiva. Método: Revisão da literatura em seis bases de dados: CINAHAL, Eric, GALE, LILACS, MEDLINE e PsycInfo. Foi utilizada a análise crítica descrita por Grant e Booth para avaliação das pesquisas localizadas. Resultados: Nenhum estudo brasileiro foi encontrado e, dentre os 29 artigos internacionais selecionados, 17 apresentavam modelos conceituais utilizados na área de T.A., sendo que, destes, 14 eram específicos de T.A. Os resultados mostraram que novos modelos conceituais de T.A. estão em desenvolvimento e que o modelo Matching Person and Technology - MPT foi o mais citado. Conclusão: Pode-se observar que as práticas envolvendo a área de T.A. no contexto internacional apresentam uma correlação com modelos teóricos e espera-se que este estudo possa contribuir para a replicação destes preceitos também no âmbito nacional.
\end{abstract}

Palavras-chave: Tecnologia Assistiva, Prática Clínica Baseada em Evidências, Reabilitação.

\section{Theoretic models for recommendation and implementation of assistive technology}

\begin{abstract}
Introduction: The latest international researches seek to understand the factors affecting the successful use of assistive technology devices through studies regarding the assessments systematizing; abandonment of devices; or theoric models that consider the aspects of those devices implementation. In Brazil the researches are focused on developing new technologies and there are still not sufficient studies related to the successful use of devices and ways of assistive technology implementation. Objective: To identify conceptual models used for indication and implementation of assistive technology devices. Method: Literature review. The survey was conducted in six databases: CINAHAL, Eric, GALE, LILACS, MEDLINE e PsycInfo. A critical analysis described by Grant and Booth was used. Results: There are no records of a Brazilian survey and among 29 selected articles, 17 conceptual models used in the area of AT were found; of these, 14 were specific to AT. The results showed that the new conceptual models of TA are under development and the conceptual model "Matching Person and Technology - MPT" was the most mentioned. Conclusion: We can observe that the practices related to TA area in international context shows a correlation with conceptual models, thus, we hope this study might have the capacity to contribute for the propagation of this precepts at national level.
\end{abstract}

Keywords: Self-help Devices, Evidence-based Practice, Rehabilitation.

Autor para correspondência: Ana Cristina de Jesus Alves, Universidade de Brasília, Campus Ceilândia, QNN 14 Área Especial, Ceilândia Sul, CEP 72220-140, Brasília, DF, Brasil, e-mail: crisjalves@yahoo.com.br

Recebido em Set. 16, 2014; $1^{\text {a }}$ Revisão em Nov. 10, 2014; Aceito em Dez. 23, 2014. 


\section{Introdução}

A tecnologia tem sido utilizada para auxiliar indivíduos com deficiências a adquirir maior autonomia e realizar tarefas do cotidiano.

Em 2006, o Comitê Brasileiro de Tecnologia Assistiva (CAT) estabelece, entre suas ações, a pesquisa para proposição de uma terminologia oficial para a definição de tecnologia que auxilie os indivíduos com deficiências e, assim, define:

TecnologiaAssistivaéumaárea do conhecimento, de característica interdisciplinar, que engloba produtos, recursos, metodologias, estratégias, práticas e serviços que objetivam promover a funcionalidade, relacionada à atividade e participação, de pessoas com deficiência, incapacidades ou mobilidade reduzida, visando sua autonomia, independência, qualidade de vida e inclusão social ${ }^{2}$ (BRASIL, 2009, p. 9).

Considerando-se as pesquisas internacionais envolvendo a área da tecnologia assistiva, os principais temas abordados são: os fatores que influenciam o uso bem sucedido dos dispositivos de tecnologia assistiva; as causas determinantes para o abandono dos dispositivos de tecnologia assistiva; a identificação de variáveis a serem consideradas na indicação e na implementação de dispositivos, e a busca de medidas de eficácia que avaliem o uso de dispositivos de T.A., dentre outros (RIEMER-REISS; WACKER, 2000; SCHERER et al., 2005; SCHERER et al., 2007).

Já na literatura nacional, predominam as publicaçóes que abordam indicaçôes de dispositivos que podem favorecer o melhor desempenho de indivíduos com deficiência, como pode ser visto nos trabalhos de Manzini e Deliberato (2006), Bersch e Pelosi (2006), Schirmer et al. (2007), Cruz e Ioshimoto (2010) e Agnelli (2012).

$\mathrm{Na}$ literatura internacional, estudos sobre implementação de dispositivos de T.A. são mais comumente encontrados. Estes seguem tanto abordagens teóricas, baseadas em formulaçôes de modelos conceituais, quanto abordagens práticas, descrevendo procedimentos de implementação (JUDGE, 2002; LENKER; PAQUET, 2003; FUHRER et al., 2003; PARETTE; BROTHERSON, 2004; LENKER; PAQUET, 2004; SCHERER et al., 2007; BERND; VAN DER PIJL; WITTE, 2009).

Em relação às formulações teóricas descritas na literatura internacional, pesquisas apontam para a importância de haver modelos teóricos que embasem a prática de implementação de T.A.
Em estudo de revisão, feito por Bernd, van der Pijl e Witte (2009), sobre os modelos teóricos e instrumentos para seleção e implementação de dispositivos de T.A., sete modelos foram apresentados, destacando-se entre estes o modelo Matching Person and Technology Model - MPT (SCHERER et al., 2005). Os autores discutem que ainda há modelos pouco sistematizados, com avaliações náo padronizadas e pouco aplicáveis à prática clínica.

Já Lenker e Paquet (2003) destacaram três modelos: o MPT; o Human-Activity Technology Model (HAT), e a Classificação Internacional de Funcionalidade, Incapacidade e Saúde (CIF), considerando-os os mais completos por classificarem e descreverem características associadas ao indivíduo e ao ambiente.

Considerando-se esses três modelos, o Matching Person and Technology - MPT (SCHERER et al., 2005) sugere que três áreas devem ser dirigidas, ao se avaliar a predisposição de um indivíduo ao uso da tecnologia, sendo estas: a) os fatores psicossociais; b) os fatores do ambiente em que o dispositivo de tecnologia assistiva será utilizado, e c) os fatores específicos da tecnologia (SCHERER et al., 2005).

O modelo descrito por Cook e Hussey enfatiza a importância da pessoa, da atividade e do contexto na escolha da tecnologia assistiva. Mudanças em qualquer um desses componentes podem exigir mudanças da tecnologia. Esse modelo conceitual mostra que a tecnologia assistiva pode vincular a pessoa à atividade e, caso a T.A. seja retirada, pode separar o indivíduo do desempenho da tarefa (COOK; POLGAR, 2008).

Já a CIF, embora não seja um modelo específico de tecnologia assistiva, tem como pressuposto unificar, de forma conceitual, a descrição de saúde e dos estados relacionados a esta. Os dispositivos de T.A. estão inseridos nesta classificação como parte dos fatores ambientais/contextuais, os quais constituem o ambiente físico, social e atitudinal em que as pessoas vivem e conduzem suas vidas, podendo estes atuar como facilitadores ou barreiras (ORGANIZAÇÃO..., 2008).

Em relação às pesquisas que focalizam as práticas de implementação de T.A., algumas são evidenciadas na literatura internacional, como, por exemplos: a Prática Baseada em Evidência em TA, a Prática Centrada no Usuário e a Prática Centrada na Família.

A Prática Baseada em Evidência em T.A., descrita por Fuhrer et al. (2003), tem como objetivos investigar e validar a contribuição e o impacto trazido pela T.A. ao indivíduo. Os autores propóem que a implementação de T.A. tenha início com a procura do dispositivo a partir da identificação da necessidade 
do indivíduo e dos tipos de dispositivos e serviços disponíveis. $\mathrm{O}$ processo de inicialização do uso do dispositivo será caracterizado, a princípio, pelo uso em curto prazo. Então, posteriormente, serão investigados a efetividade, a eficiência, a satisfação, o bem-estar e os fatores moderadores (custo, fatores corporais e ambientais), os quais determinarão a troca ou a manutenção do dispositivo. Já o uso em longo prazo terá, como fatores determinantes, os fatores moderadores.

A prática Centrada no Usuário, descrita por Lenker e Paquet (2004), propóe que a tomada de decisão em relação aos dispositivos de T.A. seja do usuário. $\mathrm{O}$ processo de implementação deve ser um processo contínuo, no qual o usuário identificará as vantagens ou desvantagens trazidas pelo dispositivo, sendo estas determinantes para gerar oportunidades pessoais e, assim, juntamente com fatores contextuais, permitir a identificação de intenção ou não de uso.

Os estudos que utilizam esta abordagem recorrem às avaliaçôes Quebec User Evaluation of Satisfaction with Assistive Technology - QUEST, Psycosocial Impacto Assistive Devices Scale - PIADS e a Medida Canadense de Desempenho Ocupacional - COPM, para avaliar os benefícios trazidos pelo dispositivo de T.A., seja através da investigaçáo da satisfaçáo do usuário, da qualidade de vida ou do desempenho de atividades cotidianas, relacionados ao uso de T.A., respectivamente.

A Prática Centrada na Família em relação à T.A. é descrita por Judge (2002) e Parette e Brotherson (2004), que propõem capacitar a família do usuário de dispositivos de T.A. - em geral, crianças - para a seleção, a tomada de decisão e o uso de dispositivo. Esta abordagem sugere que haja a investigação de demanda, tomada de decisão, identificação da necessidade do cliente e das prioridades, conhecimento da cultura, relação das características da criança com a T.A, do ambiente, das atividades naturais do usuário e dos serviços disponíveis.

Assim, diante das várias abordagens teóricas e práticas descritas na literatura internacional, e da falta de sistematização para indicação e implementaçáo de dispositivos de T.A., indicada pelas pesquisas nacionais, este estudo propóe identificar e discutir os principais modelos teóricos utilizados na área de tecnologia assistiva, segundo a literatura.

\section{Método}

Trata-se de um estudo que adota a metodologia teórico-conceitual baseada em levantamento bibliográfico da literatura.

Utilizando-se a classificação de Grant e Booth (2009), será utilizada a Revisão Crítica da Literatura, na qual, segundo os autores, o pesquisador realiza uma extensiva busca na literatura e avalia criticamente sua qualidade. Esse método vai além da mera descrição, exercendo um grau de análise e inovação conceitual. Em geral, resulta em uma hipótese ou modelo, e procura identificar itens mais significativos ao campo.

Nesta revisão, buscaram-se modelos conceituais e abordagens utilizados para a indicação e a implementação de dispositivos de T.A.

Para a busca dos artigos científicos, foram utilizadas seis bases de dados: Cumulative Index of Nursing and Allied Heath - CINAHAL, Education Resources Information Center - Eric, GALE Cengage Learning, Literatura Latino-Americana em Ciências da Saúde - LILACS, Sistema Online de Busca e Análise de Literatura Médica - MEDLINE e a base de dados da Associaçáo Americana de Psicologia - PsycInfo.

Os descritores foram combinados entre os termos relacionados à T.A. e os descritores de modelos e avaliaçôes, como mostra a Tabela 1.

O período selecionado para a consulta foi o de 2000 a 2012 e os idiomas considerados foram inglês, espanhol e português.

Foram incluídos, para a análise, os estudos que contemplaram discussões sobre modelos conceituais de T.A. Foram excluídos os artigos que focalizaram dispositivos de T.A. específicos, como, por exemplo, cadeira de rodas, entre outros, ou populaçôes específicas, como deficiente visual.

Utilizou-se a análise crítica (qualitativa), descrita por Grant e Booth (2009), que procura identificar a contribuição conceitual, incorporar a existente ou derivar uma nova teoria para a avaliação dos estudos identificados.

Foram considerados, como itens da análise qualitativa: fundamentação teórica aplicada no estudo;

Tabela 1. Descritores utilizados na pesquisa bibliográfica.

\begin{tabular}{cc}
\hline $\begin{array}{c}\text { Descritores utilizados } \\
\text { relacionados à T.A. }\end{array}$ & $\begin{array}{c}\text { Descritores utilizados relacionados } \\
\text { ao Modelo Conceitual }\end{array}$ \\
\hline Assistivedevice & Evidence-basedpratice \\
Assistivetechnology (AND) & Framework \\
Self-help device & Model \\
\hline
\end{tabular}


características descritivas; indicação de medidas de resultados; validação na literatura, e referências de utilidade para os profissionais.

\section{Resultados}

Foram encontrados 1.072 estudos, utilizando-se a combinação entre os descritores. Após a seleção dos 1.072 títulos, considerou-se que 29 respondiam aos critérios de seleção previamente estabelecidos.

$\mathrm{Na}$ análise, foram identificados 17 modelos teóricos; destes, 14 eram específicos de T.A., ou seja, modelos teóricos que foram fundamentados exclusivamente para Tecnologia Assistiva.

A seguir, nas Tabelas 2 e 3, serão apresentados os cinco modelos conceituais específicos para T.A. mais citados na literatura.

A Tabela 4 apresenta os demais modelos encontrados e suas referências.

Dentre os 17 modelos conceituais citados, 14 eram específicos de T.A. Dentre os três modelos não específicos, estáo o Perceived Attributes Theory, a CIF e a Humam Occupational Performance Pratice Integration Theory, que descrevem fundamentaçóes sobre conceitos da adaptação do indivíduo a novas situaçóes, conceito de saúde e desempenho ocupacional, respectivamente, sendo utilizados também como norteadores do uso e da implementação de T.A.

Em relação aos modelos conceituais específicos de T.A., três foram mais citados nos 29 artigos: o MPT, seguido pelo HAAT e pelo Gitlin's model. O modelo MPT foi o mais citado/ descrito, sendo apresentado em nove estudos. O HAAT e o Glitin's model foram citados com menor frequência (cinco e quatro estudos, respectivamente), e os demais modelos foram referidos em três ou menos estudos, como pode ser observado na Figura 1.

Os modelos específicos, os que apresentaram estudos de validação na literatura, foram: o MPT, CATOR, o Caregiver e o Lenker's Model.

Os modelos que apresentaram ou indicaram um instrumento de avaliaçáo foram: MPT, que sugere

Tabela 2. Principais modelos teóricos identificados a partir da Revisão da Literatura (continua).

\begin{tabular}{|c|c|c|c|c|}
\hline $\begin{array}{c}\text { Modelo } \\
\text { Conceitual }\end{array}$ & Base Conceitual & $\begin{array}{c}\text { Avaliação } \\
\text { Citada }\end{array}$ & $\begin{array}{c}\text { Validação } \\
\text { Modelo }\end{array}$ & Referências $^{1}$ \\
\hline $\begin{array}{l}\text { 1.The Matching } \\
\text { Person and } \\
\text { Technology } \\
\text { Model- MPT }\end{array}$ & $\begin{array}{l}\text { Aponta para a necessidade da } \\
\text { Combinação entre fatores do Indivíduo e } \\
\text { da Tecnologia. } \\
\text { Três elementos norteadores: } \\
\text { - Tecnologia: suas funções e } \\
\text { características; } \\
\text { - Meio social/ambiente: fatores que } \\
\text { influenciam o uso; } \\
\text { - Fatores pessoais e psicossociais: } \\
\text { considerar as necessidades preferências } \\
\text { do usuário. } \\
\text { Usa CIF como referencial. }\end{array}$ & $\begin{array}{l}\text { SOTU } \\
\text { ATD PA } \\
\text { ET PA } \\
\text { WT PA } \\
\text { HCT PA }\end{array}$ & $\begin{array}{l}\text { Sim. } \\
150 \\
\text { profissionais } \\
25 \text { estados } \\
\text { E.U.A. } \\
\text { Treinamento: } \\
\text { Curso EaD } \\
\text { Aplicação } \\
\text { ITD-PA }\end{array}$ & $\begin{array}{l}\text { Steel et al. } \\
\text { (2011), } \\
\text { Bernd, van der } \\
\text { Pijl e Witte } \\
\text { (2009), } \\
\text { Schreuer (2009), } \\
\text { Gitlow e Rakoski } \\
\text { (2009), } \\
\text { Lenker e Paquet } \\
\text { (2004), } \\
\text { Scherer (2005, } \\
\text { 2007), } \\
\text { Hersh e Johnson } \\
\text { (2008a), } \\
\text { Wielandt et al. } \\
\text { (2006) }\end{array}$ \\
\hline $\begin{array}{l}\text { 2. Human, } \\
\text { Activity and } \\
\text { Assistive } \\
\text { Technology- } \\
\text { HAAT }\end{array}$ & $\begin{array}{l}\text { A Tecnologia tem papel na relação } \\
\text { homem e atividade: contexto físico e } \\
\text { social é considerado. } \\
\text { Três elementos norteadores: } \\
\text { - Humano: atributos } \\
\text { neurocognitivos } \rightarrow \text { inputs, } \\
\text { processamento central e outputs; } \\
\text { - Atividade: o que o indivíduo gostaria } \\
\text { de realizar; } \\
\text { - T.A.: usado para superar a barreira } \\
\text { ambiental. Usa CIF como referencial. }\end{array}$ & Não & Não & $\begin{array}{l}\text { Bernd, van der } \\
\text { Pijl e Witte } \\
\text { (2009), } \\
\text { Schreuer (2009), } \\
\text { Hersh e Johnson } \\
\text { (2008b), } \\
\text { Wielandt et al. } \\
\text { (2006), } \\
\text { Lenker e Paquet } \\
\text { (2004) }\end{array}$ \\
\hline
\end{tabular}

${ }^{1}$ Referências: identificam-se aqui artigos que citaram ou discutiram os modelos, sendo apresentados pelo nome do primeiro autor e pelo ano de publicação. 
Tabela 3. Principais modelos teóricos identificados a partir da Revisão da Literatura (conclusão).

\begin{tabular}{|c|c|c|c|c|}
\hline Modelo Conceitual & Base Conceitual & $\begin{array}{c}\text { Avaliação } \\
\text { Citada }\end{array}$ & $\begin{array}{c}\text { Validação } \\
\text { Modelo }\end{array}$ & Referências $^{1}$ \\
\hline $\begin{array}{l}\text { 3. Gitlin's Model } \\
\text { for a A.T. user's } \\
\text { carer }\end{array}$ & $\begin{array}{l}\text { Modelo focado nos cuidadores de } \\
\text { usuários de T.A. } \\
\text { Considera: } \\
\text { - Diferentes estágios de independência e } \\
\text { uso de T.A. } \\
\text { - Fatores associados ao uso e não uso. }\end{array}$ & Não & Não & $\begin{array}{l}\text { Bernd, van der } \\
\text { Pijl e Witte } \\
\text { (2009), } \\
\text { Schreuer (2009), } \\
\text { Lenker e Paquet } \\
\text { (2004), } \\
\text { Fuhrer et al. } \\
\text { (2003) }\end{array}$ \\
\hline $\begin{array}{l}\text { 4. The Social } \\
\text { Cognitive Model } \\
\text { of Assistive Device }\end{array}$ & $\begin{array}{l}\text { Foca em aspectos cognitivos perceptuais } \\
\text { do usuário de T.A. } \\
\text { - Percepção do impacto da T.A.: } \\
\text { - expectativa de benefício no uso; } \\
\text { - esforço e custo da T.A.; } \\
\text { - consequência do não uso. }\end{array}$ & Não & Não & $\begin{array}{l}\text { Steel et al. (2011), } \\
\text { Bernd, van der } \\
\text { Pijl e Witte } \\
\text { (2009), } \\
\text { Lenker, Paquet } \\
(2004)\end{array}$ \\
\hline $\begin{array}{l}\text { 5. Comprehensive } \\
\text { Assistive } \\
\text { Technology-CAT }\end{array}$ & $\begin{array}{l}\text { Foca na relação tecnologia e indivíduo. } \\
\text { Três elementos norteadores: } \\
\text { - Pessoa: características, aspectos } \\
\text { sociais, atitudes; } \\
\text { - Contexto: cultural, nacional, local; } \\
\text { - Atividade: comunicação, mobilidade, } \\
\text { cognitivo, vida diária, educação e } \\
\text { trabalho, lazer. }\end{array}$ & Não & Não & $\begin{array}{l}\text { Hersh, Johnson } \\
(2008 \mathrm{a}, \mathrm{b})\end{array}$ \\
\hline
\end{tabular}

\footnotetext{
${ }^{1}$ Referências: identificam-se aqui artigos que citaram ou discutiram os modelos, sendo apresentados pelo nome do primeiro autor e pelo ano de publicação.
}

Tabela 4. Modelos encontrados na revisão bibliográfica e referências.

\begin{tabular}{|c|c|c|}
\hline Modelo Conceitual & $\begin{array}{c}\text { Especificidade } \\
\text { para a T.A. }{ }^{1}\end{array}$ & Referências ${ }^{2}$ \\
\hline $\begin{array}{l}\text { 6. Efficiency of Assistive Technology and } \\
\text { Service-EATS }\end{array}$ & Sim & $\begin{array}{l}\text { Person et al. (2002), } \\
\text { Wessels et al. (2000) }\end{array}$ \\
\hline $\begin{array}{l}\text { 7. Consortium on Assistive Technology } \\
\text { Outcomes Research (CATOR) }\end{array}$ & Sim & $\begin{array}{l}\text { Schreuer (2009), } \\
\text { Jutai et al. (2005) }\end{array}$ \\
\hline 8. Caregiver & Sim & Demers et al. (2009) \\
\hline 9. Lenker's Model & Sim & Lenker e Paquet (2004) \\
\hline 10. Ender'sModel & Sim & Enders (2000) \\
\hline $\begin{array}{l}\text { 11. Student Enviroment Tools and Tasks } \\
\text { model (SEET) }\end{array}$ & $\operatorname{Sim}$ & Gitlow e Rakoski (2009) \\
\hline 12. Decision-Makingmodel & Sim & Wielandt et al. (2006) \\
\hline 13. Bain Assistive Technology System (BATS) & Sim & Wielandt et al. (2006) \\
\hline $\begin{array}{l}\text { 14. Smith's client centred adaptative } \\
\text { equipament provision model }\end{array}$ & Sim & Wielandt et al. (2006) \\
\hline 15. The Perceived Attributes Theory & Não & $\begin{array}{l}\text { Bernd, van der Pijl e } \\
\text { Witte (2009), } \\
\text { Lenker e Paquet (2004) }\end{array}$ \\
\hline $\begin{array}{l}\text { 16. Classificação Internacional de } \\
\text { uncionalidade, Incapacidade e Saúde - CIF }\end{array}$ & Não & $\begin{array}{l}\text { Steel et al. (2011), } \\
\text { Gitlow e Rakoski (2009), } \\
\text { Hersh e Johnson (2008a), } \\
\text { Jutai et al. (2005), } \\
\text { Lenker e Paquet (2004) }\end{array}$ \\
\hline $\begin{array}{l}\text { 17. Human Occupational Performance Practice } \\
\text { Integration Theory }\end{array}$ & Não & Smith (2002) \\
\hline
\end{tabular}

${ }^{1}$ Especificidade T.A.: este item diz respeito aos modelos teóricos que fundamentaram seus conceitos exclusivamente no tema Tecnologia Assistiva. ${ }^{2}$ Referências: Identificam-se aqui artigos que citaram ou discutiram os modelos, sendo apresentados pelo nome do primeiro autor e pelo ano de publicação. 


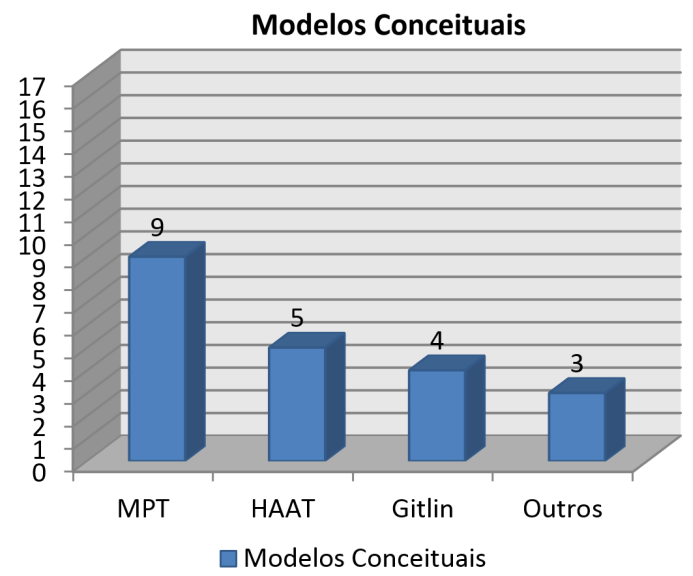

Figura 1. Modelos conceituais mais citados/ discutidos na literatura.

o uso da Survey of Technology Use - SOTU; Assistive Technology Device Presdiposition Assesssment - ATD-PA; Educational Technology Device Predisposition Assessment - ET PA; Workplace Technology Device Predisposition Assessment WT PA; Healthcare Technology Device Predisposition Assessment HCT PA; CATOR, que indica o uso da CIF, da Quebec User Evaluation of Satisfation with Assistive Technology - QUEST e da Psychosocial Impact of Assistive Device Scale - PIADS; EATS, que indica o uso da EuroQuol, e o Lenker's Model, que também sugere o uso da QUEST e da PIADS.

\section{Discussão}

A partir da análise dos resultados, pode-se apontar que nenhuma pesquisa nacional foi encontrada. Esta lacuna reforça as consideraçóes de Alves e Matsukura (2012) e Alves, Emmel e Matsukura (2012), que apontaram que, no Brasil, os estudos ainda estáo focados no desenvolvimento e na apresentação de novas tecnologias, sendo escassas as investigaçôes sobre modelos teóricos de implementação de tecnologia assistiva e avaliaçóes de eficácia.

Em relação aos investimentos teóricos em pesquisas na área, Alves, Emmel e Matsukura (2012) investigaram a formação e a prática de Terapeutas Ocupacionais que indicam dispositivos de T.A. em grandes centros de reabilitação da cidade de São Paulo, e identificaram que apenas $64 \%$ dos Terapeutas Ocupacionais participantes da pesquisa costumam publicar trabalhos na área, sendo que estas publicaçóes estáo concentradas em anais de congressos nacionais.
A partir deste contexto, hipotetiza-se que há dificuldades, por parte dos profissionais que indicam dispositivos de T.A., no contexto nacional, em fundamentar sua prática em modelos teóricos e realizar pesquisas sobre o tema.

Considerando-se os achados advindos da presente revisão, compreende-se que tal hipótese pode ser expandida aos profissionais em contexto mundial.

Embora os temas das pesquisas internacionais avancem em relação aos seus conteúdos, como a investigação de bases teóricas, as causas de abandono de T.A., os estudos de eficácia, dentre outros, ainda é escasso o número de publicaçóes internacionais, ou seja, 29 artigos num período 11 anos.

No entanto, como vimos neste estudo, novas teorias estáo em desenvolvimento e o modelo MPT foi o mais referenciado pelos estudos científicos. Por isso, o MPT foi descrito, traduzido para o português e detalhado em estudo de Alves (2013), assim como foi feita a adaptaçáo transcultural para o Brasil do instrumento de avaliação descrito pelo modelo, o ATD PA.

$\mathrm{O}$ modelo de Matching Person and Technology - o MPT - surgiu a partir de pesquisas realizadas na década de oitenta, por Scherer e Sax (2010) sobre a investigação do uso e não uso de dispositivos de T.A. por consumidores que apresentavam uma variedade de deficiências.

O modelo baseia-se na existência de três áreas cruciais, que funcionam como componentes primários na influência do uso de T.A., a saber:

a) o meio/ambiente(s): refere-se ao meio/ambiente(s) no qual o usuário irá interagir com a tecnologia, incluindo também componentes, como o apoio da família, dos pares, do empregador; o ajuste que recompense o uso do dispositivo, e a exigência externa;

b) as necessidades, preferências e predisposição individuais do usuário: incluem-se os fatores psicossociais do usuário, dentre os quais a motivação, a cooperação, o otimismo, a paciência e a autodisciplina; as experiências positivas de vida; as habilidades para o uso, e a percepçáo entre a situação desejada e a atual;

c) as funçôes e características desejáveis e apropriadas da tecnologia: estão inseridos os fatores específicos do dispositivo de tecnologia, como a habilidade de ser usado sem desconforto ou stress; a compatibilidade com outras tecnologias; o custo; a credibilidade do dispositivo; a facilidade de uso no período 
presente e futuro, e a transportabilidade (SCHERER et al., 2005, 2007; SCHERER; SAX, 2010).

Este modelo destaca que a personalidade do indivíduo e os fatores sociais influenciam o desejo ou a habilidade de uma pessoa em usar dispositivos de tecnologia assistiva. Estes componentes contribuem para uma influência positiva ou negativa no uso da T.A. e no grau de satisfação com o uso.

Segundo Scherer e Sax (2010), a satisfação em relação ao uso da T.A. é consideravelmente reduzida quanto mais influências negativas existirem. A tecnologia que pode parecer perfeita para uma necessidade pode ser usada inapropriadamente ou mesmo não ser usada, quando critérios pessoais, características sociais ou necessidades ambientais não forem consideradas, conduzindo assim a frustração pessoal e desperdício de recursos. O modelo sugere, assim, que a incompatibilidade entre a tecnologia proposta e o usuário potencial deva ser identificada precocemente, visando a reduzir o uso inadequado ou o não uso, e eliminar uma decepção e frustração, considerando-se que, em média, um terço dos dispositivos de T.A. é abandonado pelos seus usuários (SCHERER; SAX, 2010).

Desta forma, o modelo MPT foi construído a partir de um processo de avaliação, que consiste numa série de medidas que promovam a prática individualizada e centrada na pessoa, visando combinar os indivíduos com a tecnologia mais apropriada. Isto inclui um processo de avaliaçáo baseado na colaboraçáo entre o consumidor e o profissional, engajados em uma série de avaliaçóes. Estas podem incluir desde uma rápida aplicação de teste especializado (realizado em 15 minutos) até uma avaliação mais compreensiva (realizada em 45 minutos) e aplicada por um profissional treinado e experiente em seu uso. O MPT é utilizado em uma variedade de usuários e settings, e as alteraçôes do indivíduo podem ser observadas, ao longo do tempo, mediante nova aplicação das medidas de avaliação.

Scherer et al. (2005) sugerem que o foco da avaliação de um dispositivo assistivo deve passar de um modelo clínico mais tradicional para modelos que enfoquem os resultados do desempenho, tais como: a) o que acontece com o indivíduo em consequência da tecnologia, b) a qualidade de vida relatada pelos consumidores e c) avaliaçóes que forneçam resultados sobre os benefícios recebidos pelo usuário, o progresso de implementaçáo e a eficiência do recurso.
Porém, pode-se notar, com o levantamento dos estudos científicos, que outros modelos pareceram também trazer contribuiçóes importantes e ausentes no modelo MPT, como o Comprehensive Assistive Technology - CAT, criado por Hersh e Johnson (2008a).

O modelo CAT é descrito pelos autores como o mais completo, pois considera como parte da relação tecnologia e indivíduo, além dos fatores pessoais e da tecnologia, os culturais e nacionais (políticos). No entanto, neste levantamento, não foi encontrada nenhuma citação, além da pesquisa que apresentou o modelo CAT e refutou o modelo MPT, descrita pelos próprios autores (HERSH; JOHNSON, 2008b).

\section{Conclusão}

As pesquisas apresentadas reforçam a carência de métodos específicos baseados em evidências para a seleção e a implementação de T.A., principalmente no Brasil.

Nota-se que a literatura internacional tem mostrado o contínuo desenvolvimento de modelos teóricos para nortear a indicação de T.A. e que as práticas envolvendo a área de T.A., no contexto internacional, apresentam uma correlação com modelos teóricos, sendo utilizadas avaliçóes específicas, seja para a pesquisa ou para a indicaçáo de dispositivos.

Este estudo teve como pretensão disponibilizar aos profissionais, pesquisadores e demais interessados um panorama sobre a sistematização na indicação e implementação de T.A. Espera-se que este estudo possa contribuir para a replicação destes preceitos também no âmbito nacional.

\section{Referências}

AGNELLI, L. B. Avaliação da acessibilidade do idoso em sua residência. 2012. 109 f. Dissertação (Mestrado em Terapia Ocupacional) - Universidade Federal de São Carlos, São Carlos, 2012.

ALVES, A. C. J. Tecnologia assistiva: identificação de modelos e proposição de um método de implementação de recursos. 2013. 145 f. Tese (Doutorado em Educação Especial) - Universidade Federal de São Carlos, São Carlos, 2013.

ALVES, A. C. J.; EMMEL, M. L. G.; MATSUKURA, T. S. M. Formação e prática do terapeuta ocupacional que utiliza tecnologia assistiva como recurso terapêutico. Revista de Terapia Ocupacional da USP, São Paulo, v. 23, n. 1, p. 24-33, 2012.

ALVES, A. C. J.; MATSUKURA, T. S. M. O uso de recursos de tecnologia assistiva por crianças com deficiên- 
cia física na escola regular: a percepção dos professores. Cadernos de Terapia Ocupacional da UFSCar, São Carlos, v. 20, n. 3, p. 381-392, 2012.

BERND, T.; VAN DER PIJL, D.; WITTE, L. P. Existing models and instruments for the selection of assistive technology in rehabilitation practice. Scandinavian Journal of Occupational Therapy, Scandinavian, v. 16, n. 3, p. 146-158, 2009.

BERSCH, R. C. R.; PELOSI, M. B. Portal de ajudas técnicas para a educação: equipamento e material pedagógico para a educação, capacitação e recreação da pessoa com deficiência física: tecnologia assistiva: recursos de acessibilidade ao computador. Brasília: MEC, 2006.

BRASIL. Secretaria Nacional de Promoçáo dos Direitos da Pessoa com Deficiência - SNPD. Tecnologia Assistiva. Brasília: SNPD, 2009. Disponível em: <http://www.pessoacomdeficiencia.gov.br/app/publicacoes/tecnologia-assistiva>. Acesso em: 01 out. 2013.

COOK, A.; POLGAR, J. M. Cook \& Hussey's assistive technologies: principles and practice. St Louis: Mosby, 2008.

CRUZ, D. M. C.; IOSHIMOTO, M. T. A. Tecnologia assistiva para as atividades de vida diária na tetraplegia completa C6 pós-lesão medular. Revista Triângulo, Uberaba, v. 3, n. 2, p. 177-190, 2010.

DEMERS, L. et al. A conceptual framework of outcomes for caregivers of assistive technology. American Journal of Physical Medicine and Rehabilitation, Philadelphia, v. 88, n. 8 , p. 645-655, 2009.

ENDERS, A. The new paradigm of Disability: research issues and approaches conference. Washington: U. S. Department of Education, 2000.

FUHRER, M. J. et al. A framework for the conceptual modeling of assistive technology device outcomes. Disability and Rehabilitation, London, v. 25, n. 22, p. 12431251, 2003.

GITLOW, L.; RAKOSKI, M. A. Systematic assistive technology evaluation within Occupational Therapy. Occupational Therapy Practice, Bethesda, v. 14, n. 9, p. 1-8, 2009.

GRANT, M. J.; BOOTH, A. A typology of reviews: an analysis of 14 review types and associated methodologies. Health Information and Libraries Journal, Dublin, v. 26, n. 2, p. 91-108, 2009.

HERSH, M.; JOHNSON, M. A. On modeling assistive technology systems-Part I: modelling framework. Technology and Disability, Amsterdam, v. 20, n. 3, p. 251-270, 2008a.

HERSH, M.; JOHNSON, M. A. On modeling assistive technology systems-Part II: applications of comprehensive assistive technology model. Technology and Disability, Amsterdam, v. 20, n. 3, p. 251-270, 2008b.

JUDGE, B. Family-centered assistive technology assessment and intervention practices for early intervention.
Infancy Young Children, Philadelphia, v. 15, n. 1, p. 6068, 2002.

JUTAI, J. W. et al. Toward a taxonomy of assistive technology device outcomes. American Journal of Physical Medicine and Rehabilitation, Philadelphia, v. 84, n. 4, p. 294-302, 2005.

LENKER, J.; PAQUET, V. L. A review of conceptual models for assistive technology outcomes research and practice. Assistive Technology, New York, v. 15, n. 1, p. 1-15, 2003.

LENKER, J. A.; PAQUET, V. L. A review of conceptual models for assistive technology outcomes research and practice. Assistive Technology, New York, v. 16, n. 1, p. 1-10, 2004.

MANZINI, E. J.; DELIBERATO, D. Portal de ajudas técnicas para educação: equipamento e material pedagógico especial para educaçáo, capacitação e recreação da pessoa com deficiência física: recursos para comunicação alternativa. Brasília: MEC, 2006. Disponível em: $<$ http://portal.mec.gov.br/seesp/index.php?option=cont ent\&task=view\&id=157\&Itemid=309>. Acesso em: 07 jul. 2013.

ORGANIZAÇÃO MUNDIAL DA SAÚDE - OMS. Classificação internacional de funcionalidade, incapacidade e saúde. São Paulo: Universidade de São Paulo, 2008.

PARETTE, H. P.; BROTHERSON, M. J. Family-centered and culturally responsive assistive technology decision making. Infancy and Young Children, Philadelphia, v. 17, n. 4, p. 355-367, 2004.

PERSON, J. et al. Preference based assessment of quality of life of disable persons. Technology and Disability, Amsterdam, v. 14, n. 3, p. 119-124, 2002.

RIEMER-REISS, M. L.; WACKER, R. R. Factors associated with assistive technology discontinuance among individuals with disabilities. The Journal of Rehabilitation, Alexandria, v. 12, n. 2, p. 45-49, 2000.

SCHERER, M. J. et al. Predictors of assistive technology use: the importance of personal and psychosocial factors. Disability and Rehabilitation: Assistive Technology, London, v. 27, n. 21, p. 1321-1331, 2005.

SCHERER, M. et al. A framework for modeling the selection of assistive technology. Disability and Rehabilitation: Assistive Technology, London, v. 2, n. 1, p. 1-8, 2007.

SCHERER, M. J.; SAX, C. L. Measures of assistive technology predisposition and use. In: MPOFU, E.; OAKLAND, T. Rehabilitation and health assessment: applying ICF guidelines. EUA: Springer Publishing Company, 2010. p. 229-254.

SCHIRMER, C. R. et al. Atendimento Educacional Especializado: deficiência física. Brasília: MEC, 2007.

SCHREUER, N. Accommodation outcomes and the ICF framework. Assistive Technology, New York, v. 21, n. 2, p. 94-104, 2009. 
SMITH, R. O. OTFACT: multi-level performance-oriented software with an assistive technology outcomes assessment protocol. Technology and Disability, Amsterdam, v. 14, n. 3, p. 133-139, 2002.

STEEL, E. et al. Development of an AT selection tool using the CIF model. Technology and Disability, Amsterdam, v. 23, n. 1, p. 1-6, 2011.
WESSELS, L. W. et al. IPPA, a user-centred approach to assess effectiveness of assistive technology. Technology and Disability, Amsterdam, v. 13, p. 105-115, 2000.

WIELANDT, T. et al. Factors that predict the post-discharge use of recommended assistive technology. Disability and Rehabilitation: assistive technology, London, v. 1, n. 1-2, p. 29-40, 2006.

\section{Contribuição dos Autores}

Ana Cristina de Jesus Alves foi responsável pela concepção do texto, busca e organização de fontes, análises, discussão e formatação. Thelma Simóes Matsukura foi responsável pela concepção do texto, análises e discussão. Todos os autores aprovaram a versão final do texto.

\section{Fonte de Financiamento}

\section{Capes.}

\section{Notas}

${ }^{1}$ Este trabalho é parte da Tese de Doutorado da primeira autora, intitulado "Tecnologia Assistiva: identificação de modelos e proposição de um método de implementação de recursos".

${ }^{2}$ Ver Brasil (2009). 